\title{
Rapamycin improves sevoflurane-induced cognitive dysfunction in aged rats by mediating autophagy through the TLR4/MyD88/NF-kB signaling pathway
}

\author{
YAN LI, LIDAN LIU, YUE TIAN and JIN ZHANG \\ Department of Anesthesiology, Shengjing Hospital of China Medical University, Shenyang, Liaoning 110004, P.R. China
}

Received December 19, 2018; Accepted June 20, 2019

DOI: $10.3892 / \mathrm{mmr} .2019 .10541$

\begin{abstract}
The present study was aimed to observe the protective effect of rapamycin on cognitive dysfunction induced by sevoflurane in aged rats and its effect on autophagy-related proteins, and to investigate the regulatory mechanism of the Toll-like receptor $4 /$ myeloid differentiation primary response 88/nuclear factor- $\kappa \mathrm{B}$ (TLR4/MyD88/NF- $\kappa \mathrm{B}$ ) signaling pathway. Fifty Sprague-Dawley rats were randomly assigned to a control group, a sevoflurane group, a rapamycin pretreatment group, a TLR4 inhibitor group and a 3MA autophagy inhibitor group. A water maze test was used to evaluate the cognition and memory of rats. Hematoxylin and eosin (H\&E) staining was performed to observe pathological changes of brain tissue. A TUNEL assay was used to detect the apoptosis of brain tissue. ELISA was used to assess changes in brain injury markers and inflammatory factors. A western blot assay or quantitative reverse transcription PCR (RT-qPCR) were performed to determine the expression of autophagy-related proteins and the TLR4/MyD88/NF- $\mathrm{B}$ signaling pathway in brain tissue. The results revealed that rapamycin could improve cognitive dysfunction of aged rats induced by sevoflurane. Rapamycin was identified to play a therapeutic role, including mitigating brain tissue damage, inhibiting apoptosis, and activating autophagy in a sevoflurane-treated aged rat model. This function of rapamycin was demonstrated to depend on the TLR4/MyD88/NF- $\kappa \mathrm{B}$ signaling pathway.
\end{abstract}

\section{Introduction}

Postoperative cognitive dysfunction (POCD) is the impairment of certain neurophysiological regions in brain after

Correspondence to: Dr Jin Zhang, Department of Anesthesiology, Shengjing Hospital of China Medical University, 36 Sanhao Street, Shenyang, Liaoning 110004, P.R. China

E-mail: zhangj_sj@163.com

Key words: anesthesiology, rapamycin, sevoflurane, cognitive dysfunction, aged rats, autophagy, TLR4, MyD88, NF- $\mathrm{B}$, postoperative cognitive dysfunction surgical therapy, especially in older patients (1). Sevoflurane is commonly used as an inhaled anesthetic of alkanes in clinical practice (2). Sevoflurane inhalation anesthesia has been revealed to induce the increased apoptosis of hippocampal neurons in aged rats, resulting in cognitive dysfunction (3). Therefore, it is a practical and necessary task to explore the pathogenesis and mechanism of POCD, to seek effective drugs for the inhibition of neurotoxicity and cognitive dysfunction, to improve the life quality of patients after surgery, and to reduce the cost of medical treatment.

Rapamycin, a macrolide antibiotic, has been clinically used as an immunosuppressant (4). Studies have revealed that rapamycin has a protective effect on neurodegenerative diseases with strong anti-aging properties. Rapamycin can delay or inhibit the occurrence and aggravation of multiple neurodegenerative diseases (5). Therefore, rapamycin is considered to be a potential drug for the prevention and treatment of neurodegenerative diseases. Rapamycin has been revealed to improve sevoflurane-induced cognitive dysfunction in rats by activating autophagy and eliminating intracellular abnormalities and misfolded proteins (6). Researchers also identified that rapamycin-enhanced autophagy can reduce pathological changes such as amyloid- $\beta$ peptide $(\mathrm{A} \beta)$ accumulation and Tau hyperphosphorylation in the hippocampus of Alzheimer's disease (AD) rats, inhibit neuronal apoptosis and improve their learning and memory functions (7). However, the mechanism of rapamycin on improving sevoflurane-induced cognitive dysfunction in rats has not yet been elucidated.

Autophagy plays an important role in the prevention of neurodegenerative diseases, tumors and the infection of pathogenic microorganisms (8-10). Some studies have demonstrated that the occurrence and development of neurological degenerative diseases, such as AD and Parkinson's disease, are associated with autophagy dysfunction $(11,12)$. Previous studies have confirmed that the TLR4/NF- $\kappa \mathrm{B}$ signaling pathway can promote the production of reactive oxygen species in microglia and induce apoptosis (13). The present study aimed to observe the protective effect of rapamycin on sevoflurane-induced cognitive dysfunction in aged rats and its effect on autophagy-related proteins, and to explore the regulatory mechanism of the TLR4-MyD88/TRIF-NF- $\kappa$ B signaling pathway. 


\section{Materials and methods}

Experimental animals and group assignment. In total, 50 male specific-pathogen-free Sprague-Dawley rats aged 15 months and weighing 260-280 g were purchased from the Animal Laboratory Center of China Medical University (License No. SCXK(Liao)-2013-0001; License No. SYXK(Liao)-2013-0007). The project was approved by the China Medical University Institutional Animal Care and Use Committee (IACUC No. 2017018R). All animals were maintained in individual cages under controlled ambient temperature $\left(24 \pm 2^{\circ} \mathrm{C}\right)$ and $40-70 \%$ humidity in a 12 -h light/dark cycle. Standard pelleted chow and drinking water were available ad libitum. The rats were allowed to acclimate to these conditions for at least 1 week. The health and behavior of rats were observed daily by animal husbandry staff at our facility. To reduce the suffering and distress, some animal tools and nesting material were provided to the rats.

The rats were randomly assigned into five groups: i) 10 rats were placed in the blank control group (control group); ii) 10 rats were placed in the sevoflurane group (SEV group; the rats in SEV group were anesthetized for $5 \mathrm{~h}$ in $2 \%$ sevoflurane); iii) 10 rats were placed in the rapamycin pretreatment group [RAP group; the rats were intraperitoneally injected with rapamycin $(20 \mathrm{mg} / \mathrm{kg}) 2$ days before anesthesia for $5 \mathrm{~h}$ in $2 \%$ sevoflurane (14)]; iv) 10 rats were placed in the TLR4 inhibitor group [TLR group; the rats were intraperitoneally injected with rapamycin $(20 \mathrm{mg} / \mathrm{kg})$ 2 days before anesthesia for $5 \mathrm{~h}$ in $2 \%$ sevoflurane, and TLR4 inhibitor TAK242 $(0.5 \mathrm{mg} / \mathrm{kg})$ was intracerebrally injected into rats at $1 \mathrm{~h}$ before rapamycin treatment (15)]; v) 10 rats were placed in the sevoflurane+ rapamycin+3-Methyladenine (3MA) autophagy inhibitor group [3MA group; the rats in the 3MA group were intraperitoneally injected with rapamycin $(20 \mathrm{mg} / \mathrm{kg}) 2$ days before anesthesia for $5 \mathrm{~h}$ in $2 \%$ sevoflurane, and $21 \mathrm{mg} / \mathrm{kg} 3 \mathrm{MA}$ autophagy inhibitor was intracerebrally injected at $1.5 \mathrm{~h}$ before rapamycin treatment. After $24 \mathrm{~h}$, physiological data were recorded, including heart rate, mean arterial pressure, arterial carbon dioxide tension and arterial oxygen tension. After the Morris water maze (MWM) experiment, blood samples of rats were collected by saphenous vein puncture, centrifuged at $12,000 \mathrm{x} \mathrm{g}$ at $4^{\circ} \mathrm{C}$ to obtain serum and stored at $-20^{\circ} \mathrm{C}$. Then the rats were sacrificed by overdose anesthesia, $800 \mathrm{mg} / \mathrm{kg}$ pentobarbital, intraperitoneally $(6,16)$. The hippocampus of rats was collected, one half was fixed in $4 \%$ paraformaldehyde and another half was stored in liquid nitrogen.

Morris water maze experiment. The MWM experiment, consisting of an acquisition test and a special probe test, is a well-established method for assessing learning and memory abilities in rodents. The acquisition test lasted 4 days, twice daily, with 15 -minute interval between tests. On day 5, the spatial probe test was conducted. The acquisition test was performed to assess learning and memory abilities in rats. Rats were randomly placed into the water in a random order at a fixed entry point of each quadrant. The duration time to search and climb the platform in the water was recorded as the escape latency, and the rats stayed on the platform for at least $3 \mathrm{sec}$. If a rat failed to climb onto the platform within $120 \mathrm{sec}$, it was manually guided onto the platform with a stick and made to stay there for $30 \mathrm{sec}$. It was then returned to the cage for the next test. The spatial probe test was used to assess the spatial memory of rats. After the platform was removed from the pool, the rats were placed into the water from the opposite side of the original target quadrant, and allowed to search for the platform. The number of platform crossings within $120 \mathrm{sec}$ was recorded and the proportion of time spent in the original target quadrant to the total time was calculated.

$H \& E$ staining. The hippocampus fixed in paraformaldehyde was immerged in different concentrations of alcohol (70, 80, 90,95 , and $100 \%$ ), permeabilized in xylene, embedded in wax, and sliced into $4-\mu \mathrm{m}$ thick sections using a microtome. The sections were dewaxed, stained with hematoxylin for 5 min, washed with PBS, differentiated with $1 \%$ alcoholic hydrochloric acid, stained with eosin for $30 \mathrm{sec}$, followed by dehydration through a graded alcohol series and washed off. Subsequently, these sections were mounted with neutral resin. The pathological changes in the hippocampus were observed using a light microscope.

TUNEL assay. Cell apoptosis was assessed in accordance with the kit instruction (Roche Diagnostics). Briefly, 5- $\mu \mathrm{m}$ paraffin sections were dewaxed, permeabilized and sealed. After treatment with $50 \mu 1$ TUNEL reaction solution, the sections were incubated in a wet dark box at $37^{\circ} \mathrm{C}$ for $60 \mathrm{~min}$. The sections were then incubated with a NEUN antibody (1:300; cat. no. ab177487; Abcam) at $37^{\circ} \mathrm{C}$ for $1 \mathrm{~h}$. Following this, $50 \mu \mathrm{l}$ streptavidin-HRP solution and goat anti-rabbit IgG H\&L (Cy3 ${ }^{\circledR}$; 1:1,000; cat. no. ab6939; Abcam) was added followed by incubation in the dark box for $30 \mathrm{~min}$. Nuclei were stained with DAPI and observed under a fluorescence microscope. After obtaining images, the number of total nuclei and TUNEL-positive nuclei were counted. Apoptosis ratio= (the number of TUNEL-positive nuclei)/the total number of nuclei $x 100 \%$.

ELISA. ELISA was performed to assess the expression of brain injury markers S-100 $\beta$, NSE and inflammatory factors TNF- $\alpha$, IL-1 $\beta$, IL-6, and IL-10. Standard preparation (100 $\mu \mathrm{l})$ and diluted samples $(100 \mu \mathrm{l})$ were added in the corresponding reaction plate wells, lightly shaken for $30 \mathrm{sec}$, and incubated at $20-25^{\circ} \mathrm{C}$ for $20 \mathrm{~min}$. The reaction plate was washed with a washer machine. Serum sample $(100 \mu \mathrm{l})$ was added in each well and incubated at $37^{\circ} \mathrm{C}$ for $2 \mathrm{~h}$. After washing, $100 \mu \mathrm{l}$ HRP-labeled secondary antibody was added in each well and incubated at $37^{\circ} \mathrm{C}$ for $30 \mathrm{~min}$. After subsequent washing, $50 \mu \mathrm{l}$ of substrate A and $50 \mu \mathrm{l}$ of substrate B were added and visualized in a dark room for $15 \mathrm{~min}$. The reaction was terminated by adding $50 \mu \mathrm{l}$ of stop buffer. OD values were measured at $450 \mathrm{~nm}$ using a microplate reader (EXL808). The standard curve was drawn with the OD value as the ordinate and the standard concentration as the abscissa. The curve equation and $R$ value were calculated. The corresponding concentration of the sample was obtained according to the curve equation.

Western blot assay. Tissue sample was added in RIPA lysate (cat. no. 89900; Thermo Fisher Scientific, Inc.) containing a protease inhibitor on ice for $30 \mathrm{~min}$. The supernatant was collected, and the protein concentration was measured with 
a BCA Protein Quantification kit (cat. no. 23225; Thermo Fisher Scientific, Inc.). After 10\% SDS-PAGE (20 $\mu \mathrm{g}$ per lane), the samples were transferred onto PVDF membranes. Bax (1:1,000; cat. no. ab32503; Abcam), Bcl-2 (1:1,000; cat. no. ab59348; Abcam), caspase-3 (1:500; cat. no. ab13847; Abcam), Beclin1 (1:2,000; cat. no. ab207612; Abcam), LC3B (1:3,000; cat. no. ab51520; Abcam), TLR4 (1:500; cat. no. ab13556; Abcam), MyD88 (1:1,000; cat. no. ab133739; Abcam), NF-кB p65 (1:1,000; cat. no. ab246347; Abcam) and GAPDH (1:10,000; cat. no. ab181602; Abcam) antibodies were added and incubated at $4^{\circ} \mathrm{C}$ overnight. Next, the PVDF membrane was washed with PBS, a goat anti-rabbit IgG horseradish peroxidase-conjugated secondary antibody $(1: 10,000$; cat. no. ab6721; Abcam) was added and incubated at room temperature for $2 \mathrm{~h}$. The proteins were visualized with Novex ${ }^{\mathrm{TM}}$ ECL Chemiluminescent Substrate Reagent kit (Invitrogen; Thermo Fisher Scientific, Inc.) and gel imaging system (Gel Doc $^{\text {TM }}$ XR; Bio-Rad Laboratories, Inc). Absorbance values were analyzed using ImageJ (v1.8.0; National Institutes of Health).

$R T$ - $q P C R$. Brain tissue was added in TRIzol reagent (cat. no. 15596026; Invitrogen; Thermo Fisher Scientific, Inc.). According to TRIzol reagent instructions, total RNA was extracted from tissues and cells, and reversely-transcribed into first-strand cDNA (cat. no. 4387406; Invitrogen; Thermo Fisher Scientific, Inc.). In accordance with a Real-time qPCR kit (cat. no. RR820A, Takara Biotechnology Co., Ltd.), PCR was performed with the following conditions: pre-denaturation at $95^{\circ} \mathrm{C}$ for $30 \mathrm{sec} ; 40$ cycles at $95^{\circ} \mathrm{C}$ for $5 \mathrm{sec}$ and $60^{\circ} \mathrm{C}$ for $20 \mathrm{sec}$; analysis of melting curve: $95^{\circ} \mathrm{C}$ for $1 \mathrm{sec}, 65^{\circ} \mathrm{C}$ for $15 \mathrm{sec}$ and $95^{\circ} \mathrm{C}$ for $5 \mathrm{sec}$. Results were calculated using $2^{-\Delta \Delta C q}$ method (17). Primer sequences are listed in Table I.

Statistical analysis. Data were analyzed using SPSS 19.0 software (IBM Corp.), and expressed as the mean \pm standard deviation. Paired comparisons were conducted using a Student's t-test. Intergroup comparisons were analyzed using one way ANOVA followed by Tukey's post hoc test. $\mathrm{P}<0.05$ was considered to indicate a statistically significant difference.

\section{Results}

Rapamycin reduces sevoflurane-induced brain injury in aged rats. The results from $\mathrm{H} \& \mathrm{E}$ staining revealed that neurons were arranged neatly and tightly, and the cytoplasm and nucleus were plump and distinct in the control group. Neurons were arranged disorderly and loosely, cells were smaller, and nuclear condensation and cytoplasm were decreased in the SEV group. A small number of cells exhibited morphological changes, and normal neurons were still observed, which were arranged neatly and tightly in the RAP group (Fig. 1A). By ELISA detection it was revealed that compared with the control group, serum S-100 $\beta$ and NSE expression were significantly higher in the SEV group $(\mathrm{P}<0.05)$. Compared with the SEV group, serum S-100 $\beta$ and NSE expression were significantly lower in the RAP group $(\mathrm{P}<0.05$; Fig. $1 \mathrm{~B}$ and $\mathrm{C})$. These results indicated that rapamycin reduced sevoflurane-induced brain injury in aged rats.
Table I. Reverse transcription-quantitative PCR using gene primers.

\begin{tabular}{|c|c|}
\hline Gene & Primer $(5 \rightarrow 3)$ \\
\hline \multirow[t]{2}{*}{ Beclin-1 } & Forward: GACACTGGACTTCCTCCGG \\
\hline & Reverse: GATTGCTGATGTGGATAC \\
\hline \multirow[t]{3}{*}{ LC3 } & Forward: CGAGAGCGAGAGAGATGAAG \\
\hline & ACGG \\
\hline & Reverse: GGTAACGTCCCTTTTTGCCTTG \\
\hline \multicolumn{2}{|l|}{ GTA } \\
\hline \multirow[t]{2}{*}{ p62 } & Forward: CGGAGGTCATCTCAGGAAGG \\
\hline & Reverse: CGATCAGCAGAGTGGCAATAG \\
\hline \multirow[t]{2}{*}{ TLR4 } & Forward: AAGGGCTTCTACTCAGAG \\
\hline & Reverse: AGGACCCACATGGGCACT \\
\hline \multirow[t]{2}{*}{ MyD88 } & Forward: GTAGCCAGCCTCTGAAAC \\
\hline & Reverse: AGCCAGGATGATGTCTAC \\
\hline \multirow[t]{2}{*}{$\mathrm{NF}-\kappa \mathrm{B}$ p65 } & Forward: TTTCAAAAGTGGCATTGCTT \\
\hline & Reverse: TTAAGCTGTAAAATCACA \\
\hline \multirow[t]{2}{*}{ GAPDH } & Forward: GTCATCAACGGGAAACC \\
\hline & Reverse: CATGGAGAAGGCTGGGG \\
\hline
\end{tabular}

Rapamycin alleviates sevoflurane-induced cognitive dysfunction in aged rats. Models of cognitive dysfunction induced by sevoflurane inhalation in aged rats were intervened with rapamycin for $24 \mathrm{~h}$. An MWM experiment was conducted to determine the effects of rapamycin on learning and memory abilities of sevoflurane-treated rats. In the acquisition test, the escape latency of rats in each group was gradually shortened. From 2 to 4 days, compared with control group, the escape latency was significantly increased in the SEV group $(\mathrm{P}<0.05)$. Compared with the SEV group, the escape latency of the RAP group was significantly decreased $(\mathrm{P}<0.05$; Fig. $2 \mathrm{~A})$. In the spatial probe test, compared with control group, the percentage of time spent, the distance covered in the target quadrant and the number of platform crossings were significantly decreased in the SEV group $(\mathrm{P}<0.05)$. Compared with the SEV group, the percentage of time spent, the distance covered in the target quadrant and the number of platform crossings were significantly increased in the RAP group $(\mathrm{P}<0.05$; Fig. $2 \mathrm{~B})$. It is therefore indicated that rapamycin can alleviate sevoflurane-induced learning and memory impairments in aged rats.

Rapamycin mitigates sevoflurane-induced neuronal apoptosis in aged rats. TUNEL assay results revealed that compared with the control group, the number of positive cells was significantly higher in the SEV group $(\mathrm{P}<0.05)$. Compared with the SEV group, the number of positive cells in the rat hippocampus was significantly lower in the RAP group $(\mathrm{P}<0.05$; Fig. 3). Western blot assay results revealed that compared with the control group, Bcl-2 expression was significantly lower, but Bax and cleaved caspase-3 expression was significantly higher in the SEV group $(\mathrm{P}<0.05)$. Compared with the SEV group, Bcl-2 expression was significantly higher, but Bax and cleaved caspase-3 expression was significantly lower after rapamycin pretreatment in the RAP group $(\mathrm{P}<0.05$; 

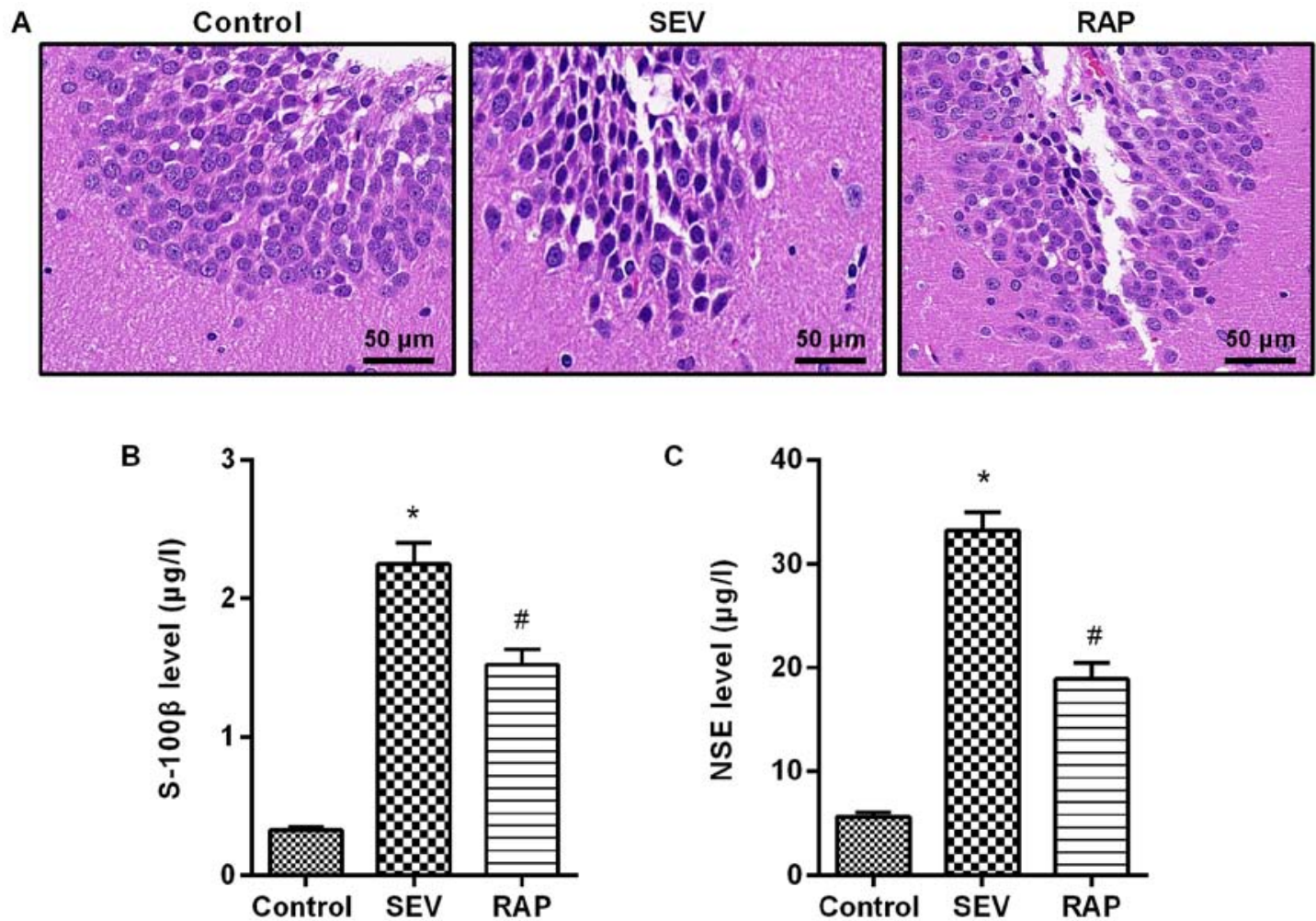

Figure 1. Rapamycin reduces sevoflurane-induced brain injury in aged rats. (A) H\&E staining (Bar, $50 \mu \mathrm{m}$ ). ELISA was performed to determine the expression levels of (B) S-100ß and (C) NSE. "P<0.05 vs. the control group; ${ }^{\text {P }}<0.05$ vs. the SEV group. H\&E, hematoxylin and eosin; SEV, sevoflurane; RAP, rapamycin.
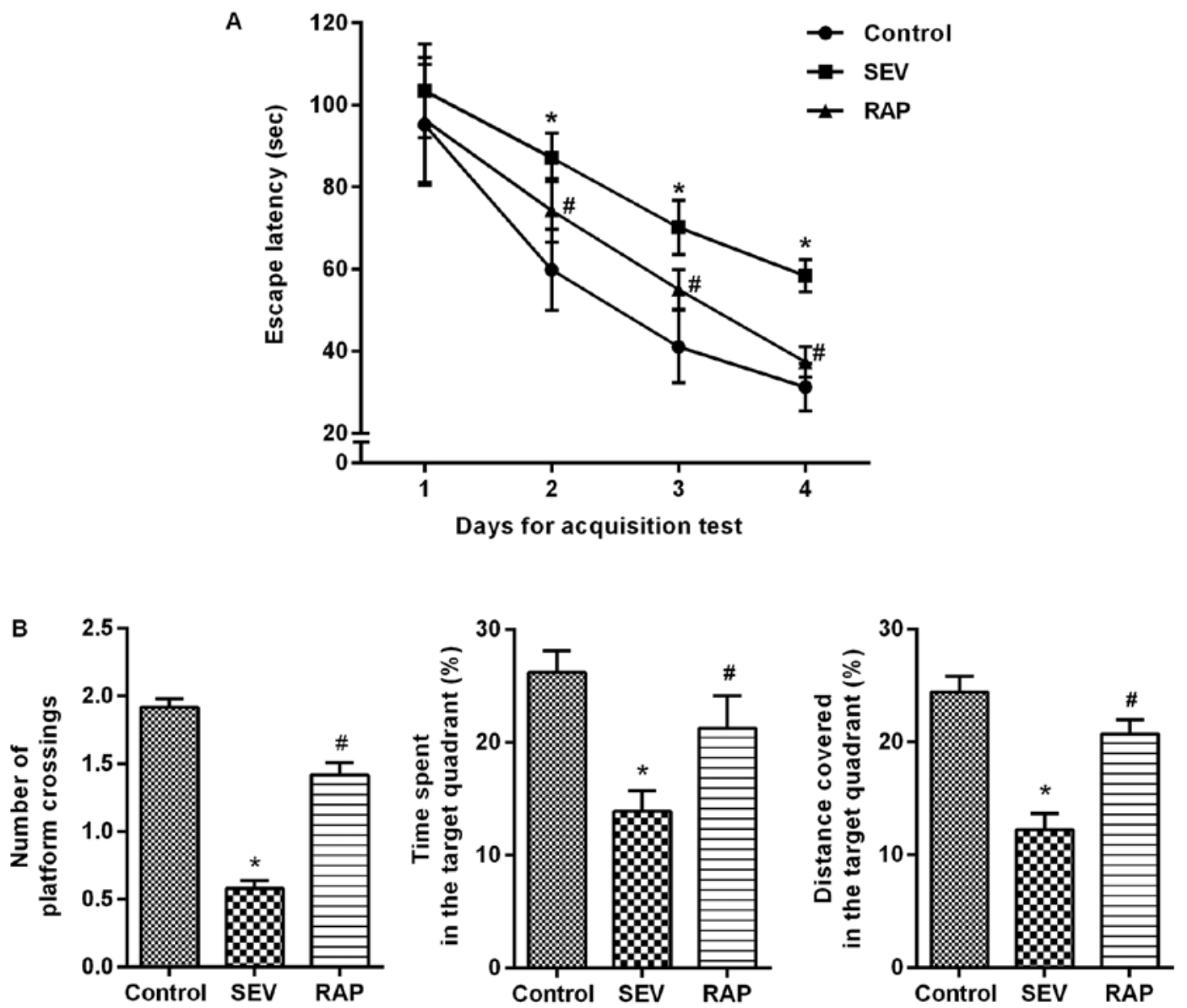

Figure 2. Rapamycin alleviates sevoflurane-induced cognitive dysfunction in aged rats. MWM test was conducted to determine the effects of rapamycin on learning and memory abilities of sevoflurane-treated rats. (A) the acquisition test; (B) the spatial probe test. " $\mathrm{P}<0.05$ vs. the control group; ${ }^{*} \mathrm{P}<0.05$ vs. the SEV group. MWM, Morris water maze; SEV, sevoflurane; RAP, rapamycin. 

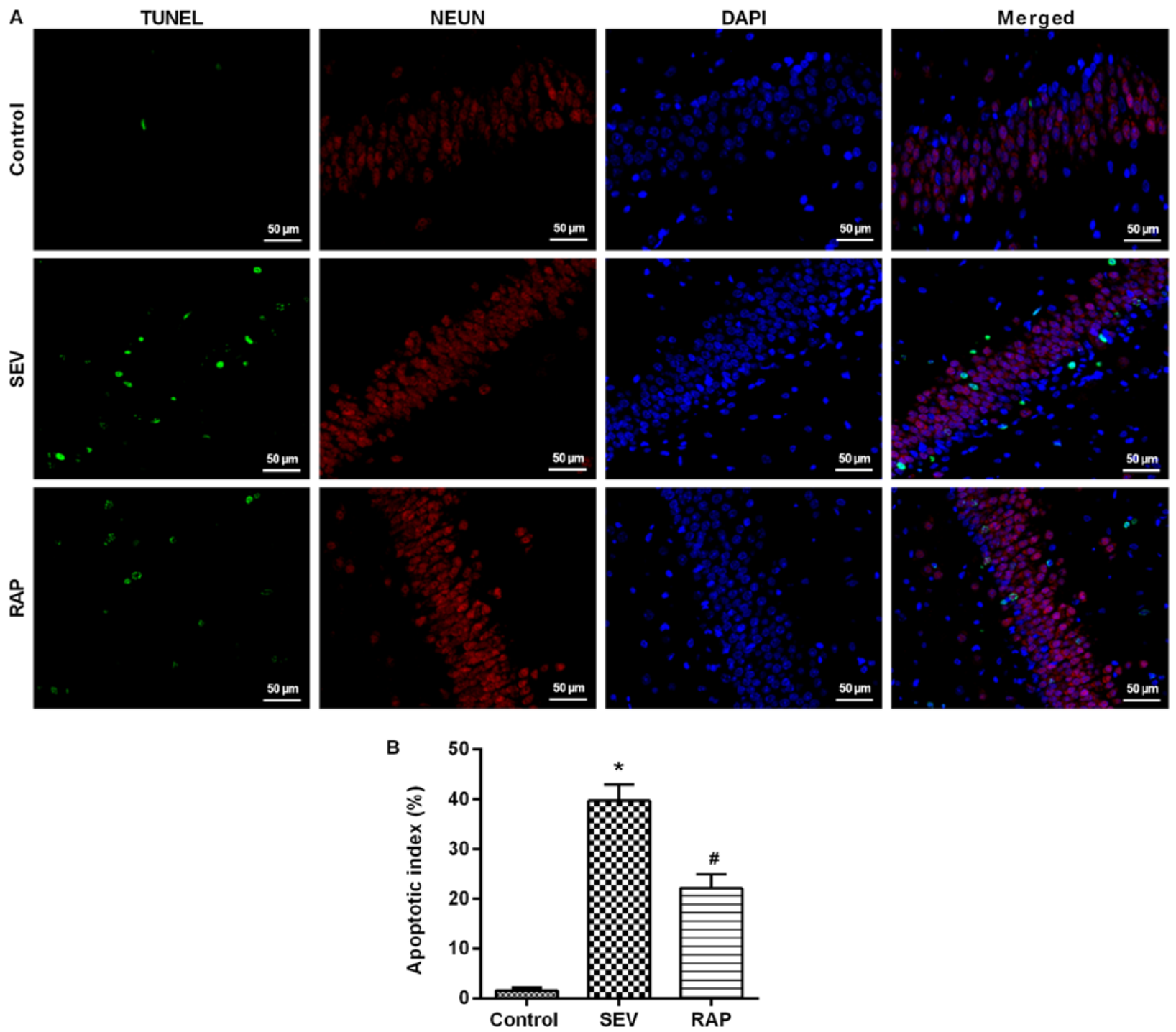

Figure 3. Rapamycin mitigates sevoflurane-induced neuronal apoptosis in aged rats. (A) Apoptosis-positive cells were determined via TUNEL assays; green, TUNEL; red, NEUN; blue, DAPI (Bar, $50 \mu \mathrm{m}$ ); (B) A bar graph of the percentage of TUNEL-positive cells. " $\mathrm{P}<0.05$ vs. the control group; ${ }^{*} \mathrm{P}<0.05$ vs. the SEV group. SEV, sevoflurane; RAP, rapamycin.

Fig. 4B). These findings indicated that rapamycin can inhibit sevoflurane-induced neuronal apoptosis and prevent neuronal degeneration.

Rapamycin reduces sevoflurane-induced inflammatory response in aged rats. ELISA results demonstrated that compared with the control group, IL-1 $\beta$, IL- 6 and TNF- $\alpha$ expression was significantly higher, but IL-10 expression was significantly lower in the SEV group $(\mathrm{P}<0.05)$. Compared with the SEV group, IL-1 $\beta$, IL- 6 and TNF- $\alpha$ expression was significantly lower, but IL-10 expression was significantly higher after pretreatment in the RAP group $(\mathrm{P}<0.05$; Fig. 4A). These results indicated that rapamycin relieves sevoflurane-induced inflammatory responses in aged rats.

Rapamycin inhibits the expression of the TLR4/MyD88/NF- $\kappa B$ signaling pathway induced by sevoflurane in aged rats. Western blot assay (Fig. 4B) and RT-qPCR (Fig. 4C) were used to determine the changes in the TLR4/MyD88/NF- $\mathrm{KB}$ signaling pathway in the rat brain. Compared with the control group, TLR4, MyD88 and NF- $\mathrm{kB}$ p65 expression was significantly higher in the SEV group $(\mathrm{P}<0.05)$. Compared with the SEV group, TLR4, MyD88 and NF-kB p65 expression was significantly lower in the RAP group $(\mathrm{P}<0.05)$. These findings indicated that rapamycin improves sevoflurane-induced brain injury in aged rats possibly by inhibiting the TLR4/MyD88/NF- $\mathrm{KB}$ signaling pathway.

Rapamycin activates sevoflurane-induced autophagy in aged rats. Western blot assay (Fig. 5A) results revealed that compared with the control group, LC3II/I and Beclin1 expression was significantly lower, but p62 expression was significantly higher in the SEV group $(\mathrm{P}<0.05)$. Compared with the SEV group, LC3II/I and Beclin1 expression was significantly higher, but p62 expression was significantly lower in the RAP group $(\mathrm{P}<0.05)$. The effect of rapamycin weakened after the administration of autophagy inhibitors. Compared with the RAP group, LC3II/I and Beclin1 expression was significantly lower, but p62 expression was significantly higher in the 3MA group $(\mathrm{P}<0.05)$. An MWM experiment was conducted to determine 


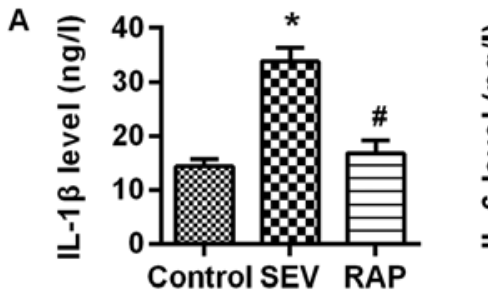

B

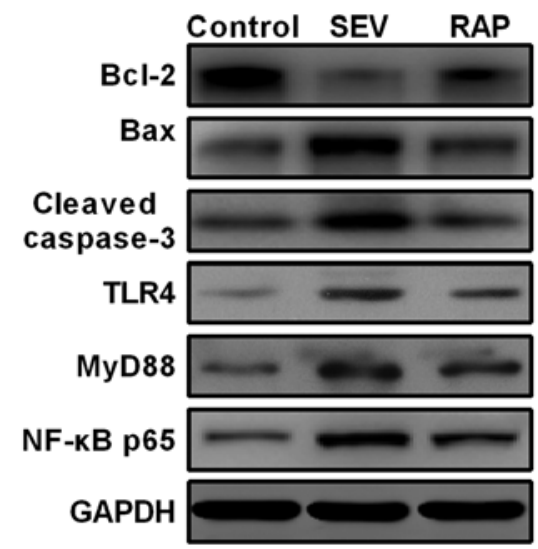

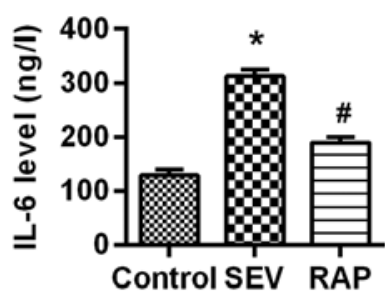
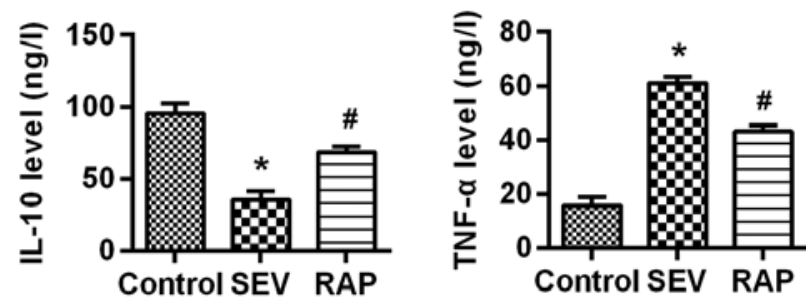

Control SEV RAP
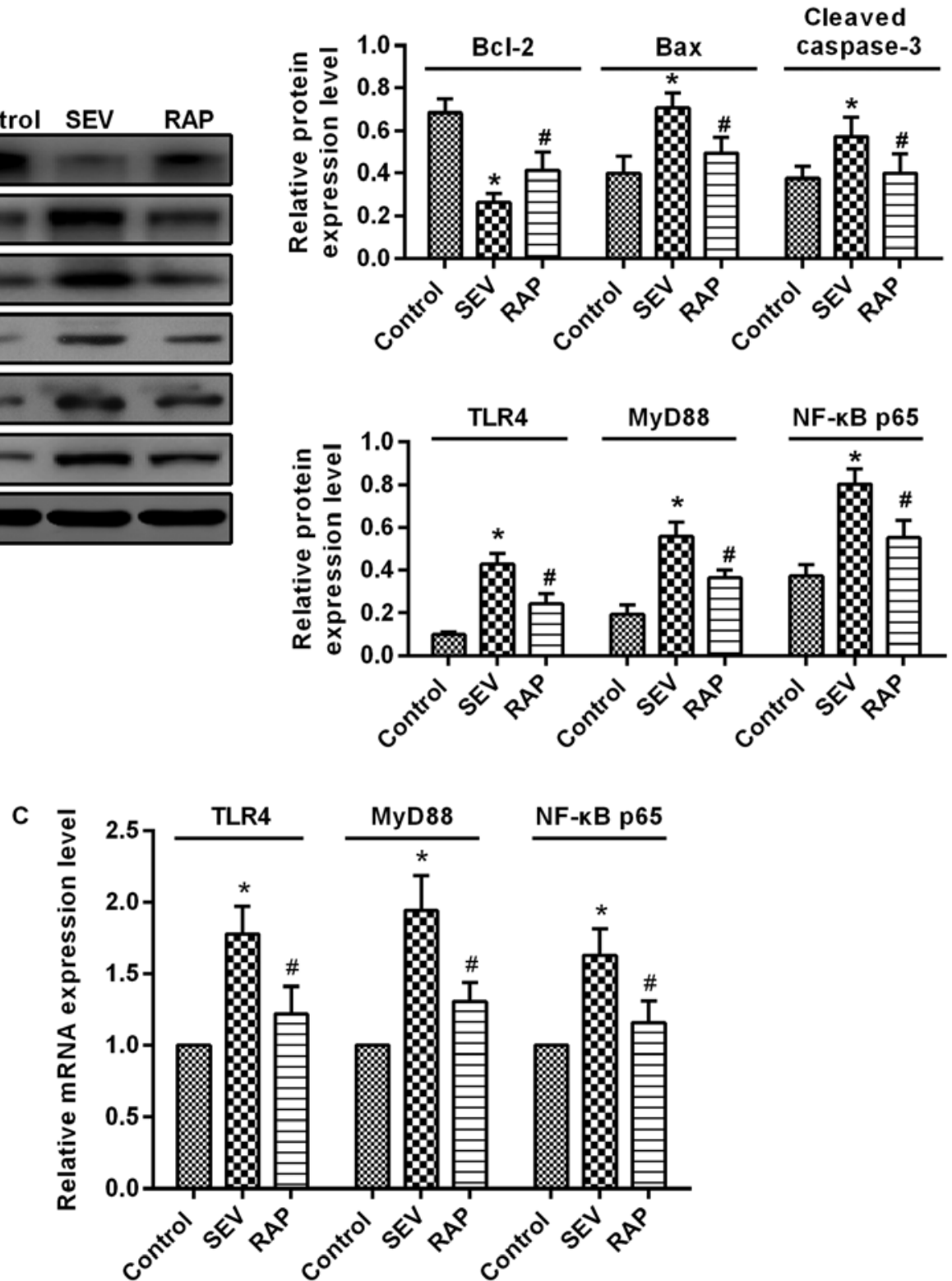

Figure 4. Rapamycin reduces sevoflurane-induced inflammatory response and inhibits the expression of the TLR4/MyD88/NF- $\mathrm{B}$ signaling pathway induced by sevoflurane in aged rats. (A) ELISA was performed to determine the expression levels of IL-1 $\beta$, IL-6, TNF- $\alpha$ and IL-10. (B) Western blot assays were performed to determine the protein expression levels of apoptosis-related proteins and TLR4/MyD88/NF-kB signaling pathway-related proteins. (C) RT-qPCR was performed to determine the expression levels of TLR4, MyD88 and NF- $\kappa B$ mRNA. ${ }^{*} \mathrm{P}<0.05$ vs. the control group; $\mathrm{P}<0.05$ vs. the SEV group. TLR4,

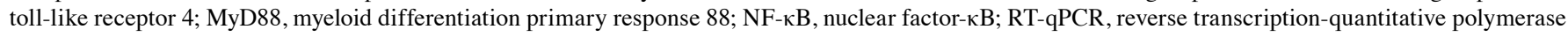
chain reaction; SEV, sevoflurane; RAP, rapamycin.

the effects of autophagy inhibitor treatment on learning and memory abilities. In the acquisition test, the escape latency of rats in each group was gradually shortened, and in 3-4 days, compared with the RAP group, the escape latency was significantly increased in the 3MA group $(\mathrm{P}<0.05)$. In the spatial probe test, compared with the RAP group, the percentage of time spent, the distance covered in the target quadrant and the number of platform crossings were significantly decreased in the 3MA group $\left(\mathrm{P}<0.05^{\prime}\right.$ Fig. $\left.5 \mathrm{~B}\right)$. These results confirmed that rapamycin activates sevoflurane-induced autophagy in aged rats.

Rapamycin improves sevoflurane-induced cognitive dysfunction in aged rats by mediating autophagy through 

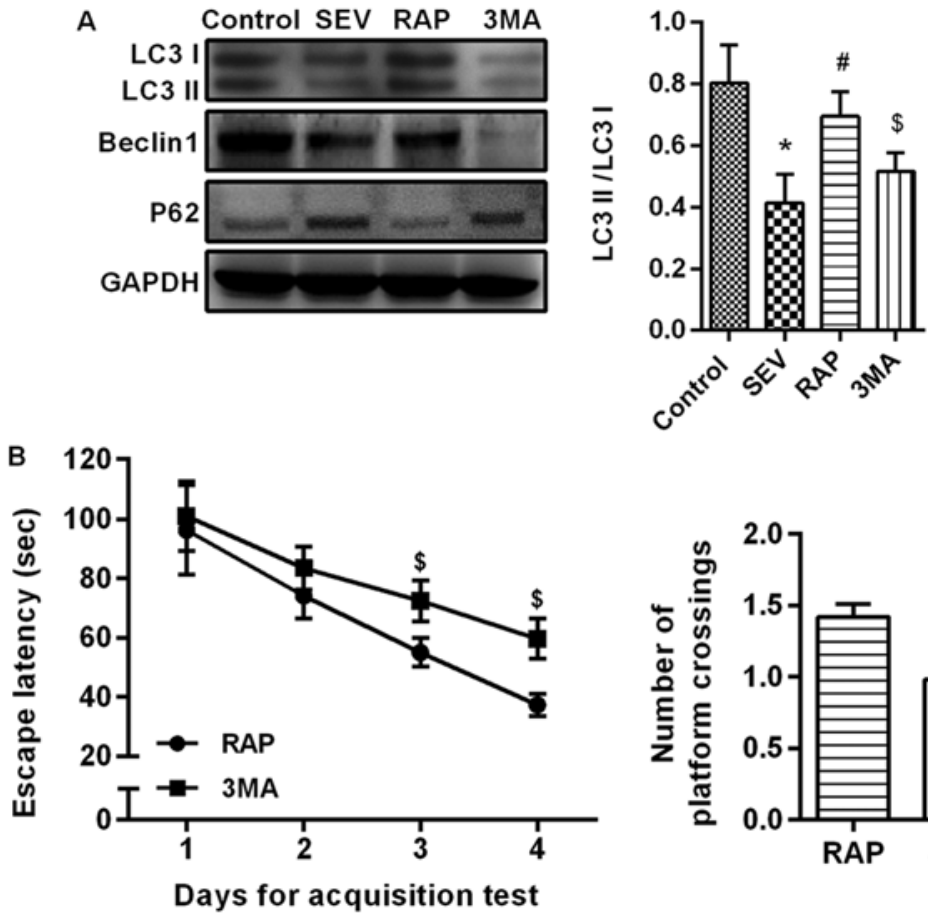
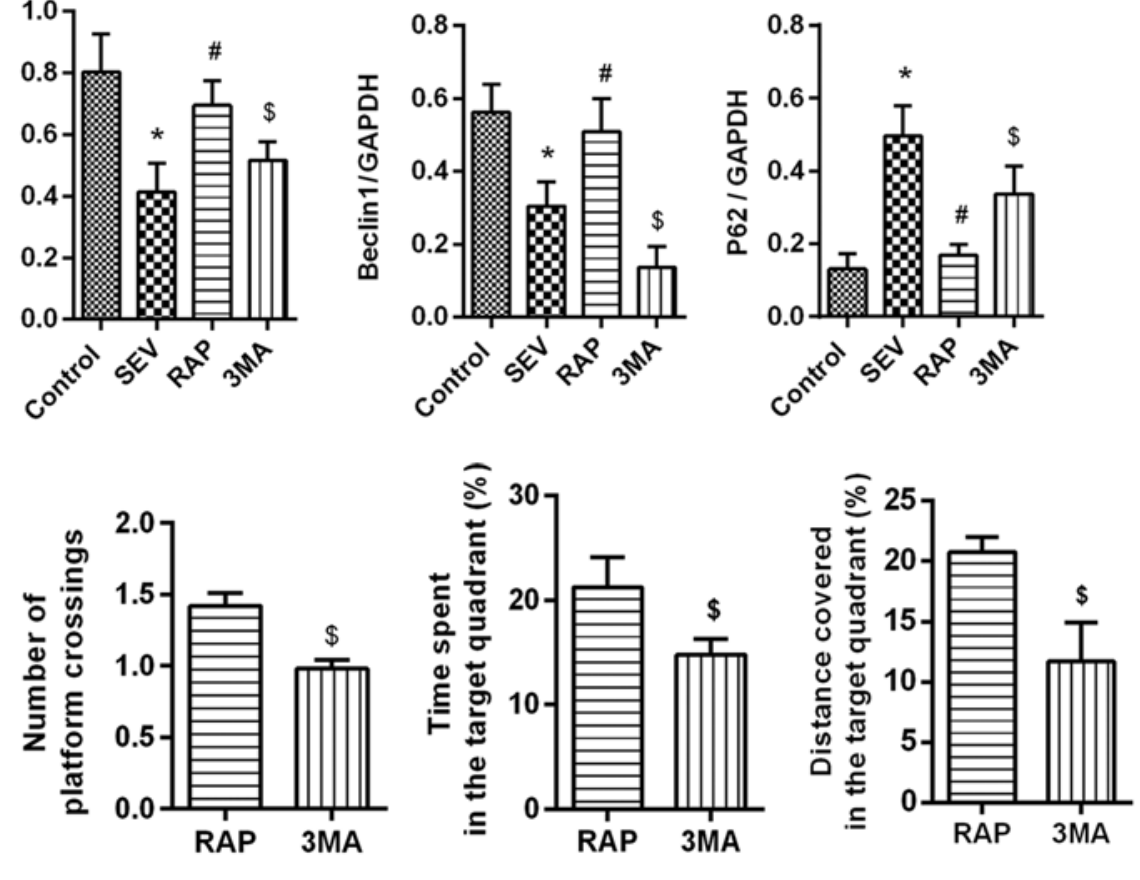

Figure 5. Rapamycin activates sevoflurane-induced autophagy in aged rats. (A) Western blot assays were performed to determine the protein expression levels of LC3II/I, Beclin1 and p62 proteins. (B) An MWM experiment was conducted to determine the effects of autophagy inhibitor treatment on learning and memory abilities. ${ }^{*} \mathrm{P}<0.05$ vs. the control group; ${ }^{\#} \mathrm{P}<0.05$ vs. the SEV group; ${ }^{\$} \mathrm{P}<0.05$ vs. the RAP group. MWM, Morris water maze; SEV, sevoflurane; RAP, rapamycin; 3MA, 3-methyladenine.

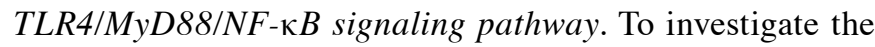
relationship between rapamycin-activated autophagy and the TLR4/MyD88/NF- $\kappa$ B signaling pathway, the aged rats with sevoflurane-induced cognitive dysfunction were treated with TLR4 inhibitor. It was revealed that compared with the RAP group, LC3II/I and Beclin1 expression was significantly lower, but p62 expression was significantly higher after suppressing the TLR4 signaling pathway $(\mathrm{P}<0.05$; Fig. 6A). ELISA was further applied to detect inflammatory factors, and as a result, the inhibitory effect of rapamycin on inflammatory factors was weakened. Compared with the RAP group, IL-1 $\beta$, IL-6 and TNF- $\alpha$ expression was significantly higher, but IL-10 expression was significantly lower in the TLR group $(\mathrm{P}<0.05$; Fig. 6B). An MWM experiment was conducted to determine the effects of the TLR4/MyD88/NF- $\kappa \mathrm{B}$ signaling pathway on learning and memory abilities. In the acquisition test, the escape latency of rats in each group was gradually shortened, and in 3-4 days, compared with the RAP group, the escape latency was significantly increased in the TLR group $(\mathrm{P}<0.05)$. In the spatial probe test, compared with the RAP group, the percentage of time, the distance covered in the target quadrant and the number of platform crossings were significantly decreased in the TLR group $(\mathrm{P}<0.05$; Fig. 6C). These findings indicated that by inhibiting the TLR4 signaling pathway, the effect of rapamycin on the activation of autophagy was weakened, and the protective effect of rapamycin on sevoflurane-induced brain injury was markedly weakened in the aged rats. These results confirmed that rapamycin improves sevoflurane-induced cognitive dysfunction in aged rats by mediating autophagy via the TLR4/MyD88/NF- $\kappa \mathrm{B}$ signaling pathway.

\section{Discussion}

POCD is a reversible and fluctuating acute neurological disorder that occurs shortly after surgery (18). POCD is mainly manifested as postoperative mental disorder, anxiety, personality changes and impaired memory (19). Some studies have revealed that age was an independent risk factor for POCD $(20,21)$. The incidence of POCD was as high as $25 \%$ within 1 week after non-cardiac surgery in elderly patients older than 60 years of age, and the symptoms of $10 \%$ of the patients lasted until three months after surgery (22). The occurrence of POCD can delay the postoperative recovery, prolong the length of hospital stay and increase medical expenses in elderly patients, and even have a certain impact on their long-term quality of life, which increases the economic burden on families and society (23). In the present study, the models of sevoflurane-induced cognitive dysfunction in the elderly in vitro and in vivo were established, and the protective effect of rapamycin was observed. The present results demonstrated that rapamycin can improve learning and memory impairments in rats, reduce the expression of inflammatory factors in rat serum, decrease apoptosis of brain tissue, and activate autophagy in tissues. This mechanism of action was exerted through the TLR4/MyD88/NF- $\mathrm{B}$ signaling pathway.

Rapamycin is used in the clinical treatment of organ transplant rejection and certain cardiovascular diseases (4). Rapamycin has a therapeutic effect on neurodegenerative diseases, such as the neuroprotective effect on both Alzheimer's disease and Parkinson's disease $(24,25)$. Neurodegenerative diseases in old age are strongly associated with age (26). Rapamycin has strong anti-aging effects and 

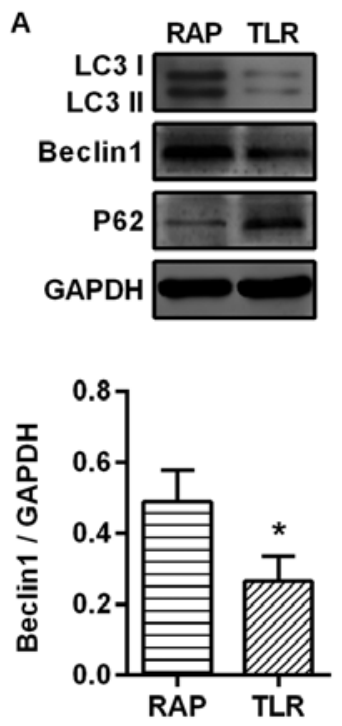
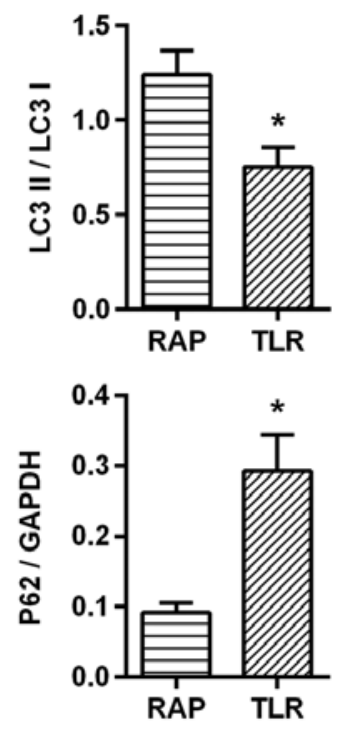
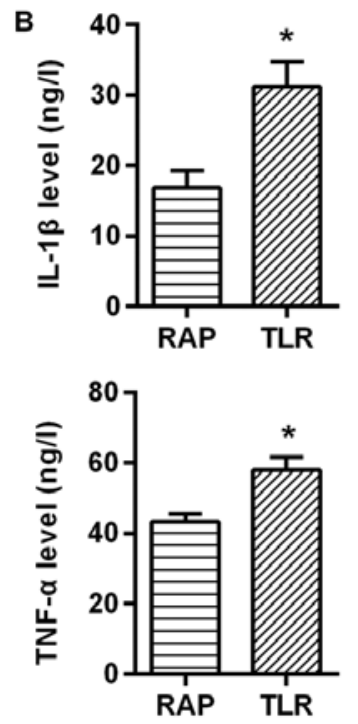
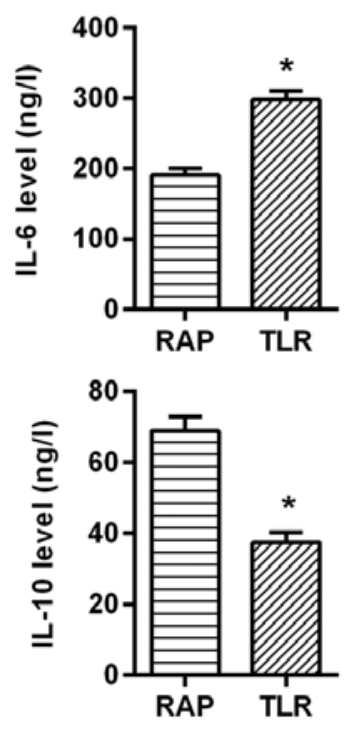

C
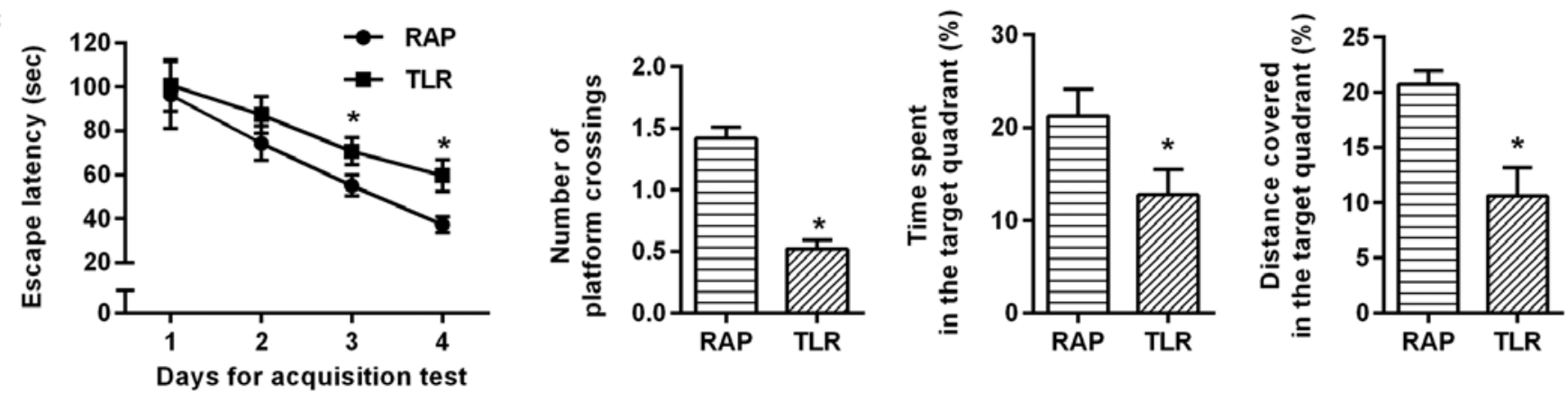

Figure 6. Rapamycin improves sevoflurane-induced cognitive dysfunction in aged rats by mediating autophagy through the TLR4/MyD88/NF-кB signaling pathway. (A) Western blot assays were performed to determine the protein expression levels of LC3II/I, Beclin1 and p62 proteins. (B) ELISA was performed to determine the expression levels of IL-1 $\beta$, IL-6, TNF- $\alpha$ and IL-10. (C) An MWM experiment was conducted to determine the effects of the TLR4/MyD88/NF- $\kappa B$ signaling pathway on learning and memory abilities. "P $<0.05$ vs. the RAP group. TLR4, toll-like receptor 4; MyD88, myeloid differentiation primary response

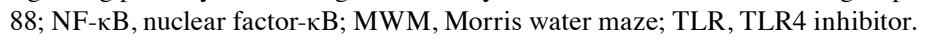

can delay the onset of such neurodegenerative diseases by altering age-related protein molecules (27). Moreover, studies have revealed that rapamycin can induce lysosome-mediated autophagy, enhance the ability of lysosomes to clear autophagosomes, and reduce autophagic accumulation, thereby playing a neuroprotective role (28). By establishing an aging cognitive dysfunction model, it was revealed that after rapamycin pretreatment in rats, learning and memory impairments were reduced, and the integrity of the brain tissue was markedly less than that of the sevoflurane group; the arrangement of neurons in the brain was neat and tight than that of the sevoflurane group; the expression of S-100 $\beta$ and NSE, which is a sign of brain tissue damage in serum, was markedly decreased, and the expression of IL-6, IL-1 $\beta$ and TNF- $\alpha$ proinflammatory cytokines was significantly decreased, while the expression of anti-inflammatory cytokine IL-10 was markedly increased; the expression of BAX and caspase- 3 in the tissue was markedly reduced, however the expression of anti-apoptotic factor Bcl-2 was significantly increased. These findings indicated that rapamycin exerts a protective effect on sevoflurane-induced cognitive dysfunction both in vitro and in vivo.

Autophagy is an important mechanism for eukaryotic cells to maintain homeostasis and survival (29). In terms of neurological diseases, autophagy is another neuronal death mode in addition to apoptosis and necrosis (30). Autophagy plays roles in different diseases and plays a dual role in neuroprotection or neuronal death $(31,32)$. Studies have revealed that TLR4 is an environmental receptor for autophagy and macrophages can induce autophagy and autophagy-mediated cell death by lipopolysaccharide (33). It was revealed that lipopolysaccharide induces autophagy through the TRIF-dependent, MyD88-independent TLR4 signaling pathway. Although there is in fact a link between TLR-mediated innate immunity and autophagy, the mechanism is still unclear. Studies have revealed that lipopolysaccharide can induce autophagy of tubular epithelial cells via the TLR4 pathway both in vivo and in vitro in acute kidney injury test, counteracting endotoxin-induced renal injury, and regulating the TLR4 downstream signaling pathway (34). The level of autophagy was suppressed in the isoprenaline-induced myocardial fibrosis model in TLR4 knockout mice (35). In the present study, it was revealed that when the TLR4/MyD88/NF- $\kappa B$ signaling pathway was inhibited, the expression of LC3-II/I and Beclin1 was significantly decreased, and the protective effect of rapamycin was markedly weakened in older models of cognitive dysfunction. It was further revealed that the expression of TLR4, MyD88, 
and NF- $\mathrm{B}$ p 65 was increased compared with the rapamycin group. These data indicated that this protective effect of rapamycin is achieved by mediating autophagy through the TLR4/MyD88/NF-кB p65 signaling pathway.

In conclusion, rapamycin can reduce learning and memory impairments and inflammatory response caused by cognitive dysfunction in the elderly in vitro and in vivo, inhibit tissue and cell apoptosis, activate tissue and cell autophagy, and alter the expression of TLR4, MyD88, and NF- $\kappa \mathrm{B}$ p65. These results demonstrated that rapamycin has an ameliorating effect on sevoflurane-induced cognitive dysfunction in older age, and this effect is achieved by mediating autophagy through the TLR4/MyD88/NF-кB p65 signaling pathway.

\section{Acknowledgements}

Not applicable.

\section{Funding}

The present study was supported by the Shenyang Key Technology Research and Development plan (grant no. 17-230-9-45).

\section{Availability of data and materials}

The datasets used and/or analyzed during the present study are available from the corresponding author on reasonable request.

\section{Authors' contributions}

YL and JZ conceived and designed the study and drafted the manuscript. YL, LL and YT performed experiments and interpreted the results. YT and JZ analyzed the data. YT and JZ contributed to acquisition of funding support. All authors read and approved the final manuscript.

\section{Ethics approval and consent to participate}

The project was approved by the China Medical University Institutional Animal Care and Use Committee (IACUC no. 2017018R).

\section{Patient consent for publication}

Not applicable.

\section{Competing interests}

The authors declare that they have no competing interests.

\section{References}

1. Benhamou D and Brouquet A: Postoperative cerebral dysfunction in the elderly: Diagnosis and prophylaxis. J Visc Surg 153: S27-S32, 2016.

2. Brioni JD, Varughese S, Ahmed R and Bein B: A clinical review of inhalation anesthesia with sevoflurane: From early research to emerging topics. J Anesth 31: 764-778, 2017.

3. Huang L, Huang K and Ning H: Autophagy induction by hispidulin provides protection against sevoflurane-induced neuronal apoptosis in aged rats. Biomed Pharmacother 98: 460-468, 2018.
4. Yoo YJ, Kim H, Park SR and Yoon YJ: An overview of rapamycin: From discovery to future perspectives. J Ind Microbiol Biotechnol 44: 537-553, 2017.

5. Maiese K: The mechanistic target of rapamycin (mTOR) and the silent mating-type information regulation 2 homolog 1 (SIRT1): Oversight for neurodegenerative disorders. Biochem Soc Trans 46: 351-360, 2018.

6. Zhang X, Zhou Y, Xu M and Chen G: Autophagy Is Involved in the Sevoflurane Anesthesia-Induced Cognitive Dysfunction of Aged Rats. PLoS One 11: e0153505, 2016.

7. Cai Z, Zhao B, Li K, Zhang L, Li C, Quazi SH and Tan Y: Mammalian target of rapamycin: A valid therapeutic target through the autophagy pathway for Alzheimer's disease? J Neurosci Res 90: 1105-1118, 2012.

8. Nah J, Yuan J and Jung YK: Autophagy in neurodegenerative diseases: From mechanism to therapeutic approach. Mol Cells 38: 381-389, 2015.

9. Gewirtz DA: The four faces of autophagy: Implications for cancer therapy. Cancer Res 74: 647-651, 2014.

10. Espert L, Beaumelle B and Vergne I: Autophagy in Mycobacterium tuberculosis and HIV infections. Front Cell Infect Microbiol 5: 49, 2015.

11. Ntsapi C, Lumkwana D, Swart C, du Toit A and Loos B: New insights into autophagy dysfunction related to amyloid beta toxicity and neuropathology in alzheimer's disease. Int Rev Cell Mol Biol 336: 321-361, 2018.

12. Segura-Aguilar J and Huenchuguala S: Aminochrome Induces Irreversible Mitochondrial Dysfunction by Inducing Autophagy Dysfunction in Parkinson's Disease. Front Neurosci 12: 106, 2018.

13. Wang Z, Liu D, Wang F, Liu S, Zhao S, Ling EA and Hao A: Saturated fatty acids activate microglia via Toll-like receptor 4/NF- $\kappa \mathrm{B}$ signalling. Br J Nutr 107: 229-241, 2012.

14. Chi OZ, Mellender SJ, Barsoum S, Liu X, Damito S and Weiss HR: Effects of rapamycin pretreatment on blood-brain barrier disruption in cerebral ischemia-reperfusion. Neurosci Lett 620: 132-136, 2016.

15. Feng Y, Gao J, Cui Y,Li M,Li R, Cui C and Cui J: Neuroprotective effects of resatorvid against traumatic brain injury in rat: Involvement of neuronal autophagy and TLR4 signaling pathway. Cell Mol Neurobiol 37: 155-168, 2017.

16. Zatroch KK, Knight CG, Reimer JN and Pang DS: Refinement of intraperitoneal injection of sodium pentobarbital for euthanasia in laboratory rats (Rattus norvegicus). BMC Vet Res 13: 60, 2017.

17. Livak KJ and Schmittgen TD: Analysis of relative gene expression data using real-time quantitative PCR and the 2(-Delta Delta C(T)) Method. Methods 25: 402-408, 2001.

18. Rundshagen I: Postoperative cognitive dysfunction. Dtsch Arztebl Int 111: 119-125, 2014.

19. Berger M, Nadler JW, Browndyke J, Terrando N, Ponnusamy V, Cohen HJ, Whitson HE and Mathew JP: Postoperative cognitive dysfunction: Minding the gaps in our knowledge of a common postoperative complication in the elderly. Anesthesiol Clin 33: 517-550, 2015.

20. Tang Y, Wang X, Zhang S, Duan S, Qing W, Chen G, Ye F, Le Y and Ouyang W: Pre-existing weakness is critical for the occurrence of postoperative cognitive dysfunction in mice of the same age. PLoS One 12: e0182471, 2017.

21. Li Z, Liu F, Ma H, White PF, Yumul R, Jiang Y, Wang N and Cao X: Age exacerbates surgery-induced cognitive impairment and neuroinflammation in sprague-dawley rats: The role of IL-4. Brain Res 1665: 65-73, 2017.

22. Coburn M, Fahlenkamp A, Zoremba N and Schaelte G: Postoperative cognitive dysfunction: Incidence and prophylaxis. Anaesthesist 59: 177-184; quiz 185, 2010.

23. Silbert B, Evered L, Scott DA, McMahon S, Choong P, Ames D, Maruff $\mathrm{P}$ and Jamrozik K: Preexisting cognitive impairment is associated with postoperative cognitive dysfunction after hip joint replacement surgery. Anesthesiology 122: 1224-1234, 2015.

24. Li L: The molecular mechanism of glucagon-like peptide-1 Therapy in Alzheimer's disease, based on a mechanistic target of rapamycin pathway. CNS Drugs 31: 535-549, 2017.

25. Jiang J, Jiang J, Zuo Y and Gu Z: Rapamycin protects the mitochondria against oxidative stress and apoptosis in a rat model of Parkinson's disease. Int J Mol Med 31: 825-832, 2013.

26. Jove M, Portero-Otin M, Naudi A, Ferrer I and Pamplona R: Metabolomics of human brain aging and age-related neurodegenerative diseases. J Neuropathol Exp Neurol 73: 640-657, 2014.

27. Krzesniak M,Zajkowicz A,Matuszczyk I and Rusin M: Rapamycin prevents strong phosphorylation of p53 on serine 46 and attenuates activation of the p53 pathway in A549 lung cancer cells exposed to actinomycin D. Mech Ageing Dev 139: 11-21, 2014. 
28. Wang Y, Ma Q, Ma X, Zhang Z, Liu N and Wang M: Role of mammalian target of rapamycin signaling in autophagy and the neurodegenerative process using a senescence accelerated mouse-prone 8 model. Exp Ther Med 14: 1051-1057, 2017.

29. Parzych KR and Klionsky DJ: An overview of autophagy: Morphology, mechanism, and regulation. Antioxid Redox Signal 20: 460-473, 2014.

30. Twayana KS and Ravanan P: Eukaryotic cell survival mechanisms: Disease relevance and therapeutic intervention. Life Sci 205: 73-90, 2018.

31. Saha S, Panigrahi DP, Patil S and Bhutia SK: Autophagy in health and disease: A comprehensive review. Biomed Pharmacother 104: 485-495, 2018

32. Luo T, Liu G, Ma H, Lu B, Xu H, Wang Y, Wu J, Ge P and Liang J: Inhibition of autophagy via activation of PI3K/Akt pathway contributes to the protection of ginsenoside $\mathrm{Rb} 1$ against neuronal death caused by ischemic insults. Int J Mol Sci 15: 15426-15442, 2014.
33. Chen S, Yuan J, Yao S, Jin Y, Chen G, Tian W, Xi J, Xu Z, Weng D and Chen J: Lipopolysaccharides may aggravate apoptosis through accumulation of autophagosomes in alveolar macrophages of human silicosis. Autophagy 11: 2346-2357, 2015.

34. Nair AR, Masson GS, Ebenezer PJ, Del Piero F and Francis J: Role of TLR4 in lipopolysaccharide-induced acute kidney injury: protection by blueberry. Free Radic Biol Med 71: 16-25, 2014.

35. Dong RQ, Wang ZF, Zhao C, Gu HR, Hu ZW, Xie J and Wu YQ: Toll-like receptor 4 knockout protects against isoproterenol-induced cardiac fibrosis: The role of autophagy. J Cardiovasc Pharmacol Ther_20: 84-92, 2015.

This work is licensed under a Creative Commons Attribution-NonCommercial-NoDerivatives 4.0 International (CC BY-NC-ND 4.0) License. 